\title{
The intermittent punishment effect: A replication with improved controls'
}

\author{
R. M. KINLER and R. K. BANKS, \\ University of Waterloo, Waterloo, Ont., \\ Canada
}

Intermittent punishment (IP) of an instrumental response in rats increased persistence to continuous punishment when $I P$ and control Ss were equated (via a goal-placement procedure) for frequency, intensity, duration, and locus of aversive stimulation prior to continuous punishment. The results were interpreted as inconsistent with adaptation interpretations of the intermittent punishment effect and as favoring a conflict resolution model.

Intermittent punishment (IP) of an instrumental response increases the persistence of that response to continuous punishment (CP) (Banks, 1966a; Brown \& Wagner, 1964; Miller, 1960; Terris \& Wechkin, 1967). This effect, termed an intermittent punishment effect (IPE), has been viewed as analogous to the partial reinforcement effect and similar leamingbased interpretations have been suggested for both phenomena. While a learning interpretation of the IPE may be appropriate, the effect appears open to a simple adaptation approach. In the typical IPE experiment (e.g., Brown \& Wagner; Terris \& Wechkin), IP Ss receive mild and infrequent aversive stimulation prior to $\mathrm{CP}$. Control Ss, on the other hand, encounter aversive stimulation for the first time with the onset of CP. Thus IP and control Ss are provided with differential opportunities to adapt to aversive stimulation.

In experiments attempting to equate adaptation, controls have received prior aversive stimulation, via placement, in a distinctive situation temporally and spatially removed from the experimental situation. Under this procedure the IPE continues to be observed (Miller, 1960) but its magnitude is reduced (Banks, 1966b), indicating that some adaptation may occur in the IPE experiment. The present study was prompted by the possibility that adaptation is to some extent specific to the situation in which aversive stimulation is received. Accordingly, the experiment was conducted so that control Ss received prior aversive stimulation, via placement, in the same situation in which IP Ss received intermittent punishment. Thus the groups were equated for frequency, intensity, duration,

\section{and locus of prior aversive stimula tion.} METHOD

Eighteen male albino rats of the Hol tzman strain, approximately 90 days old at the beginning of the experiment, served as Ss. They were randomly assigned to an IP and a control group.

The apparatus consisted of a $5-\mathrm{ft}$ alley, 4 in. high and 5 in. wide. The alley had a grid floor and was equipped with start and goal doors. Raising the start door activated a recording timer which stopped when $S$ broke a photobeam 1 in. in front of a circular food cup, $1 \mathrm{in}$. high and $1 \frac{1}{2} \mathrm{in}$. in diam, placed at the end of the 11-in. goal box.

Following 4 days of $22 \frac{1}{2}-\mathrm{h}$ food deprivation, all animals were given 2 days of habituation to the alley, with all doors raised, and free access to $.045-\mathrm{g}$ Noyes pellets in the goal cup. Training consisted of 8 days of 10 trials each with five pellets present on each trial. A 30 -sec intertrial interval, during which $\mathbf{S}$ was placed on a waiting stand, was employed during training. Differential treatment consisted of 10 trials per day for 10 days. On three trials of each day controls received nonreward and, on the same three trials, IP Ss received nonreward and punishment. Punishment consisted of a $.1-\mathrm{sec}$ shock of $.25 \mathrm{~mA}$, delivered to the grid floor of the goal box $.5 \mathrm{sec}$ after the photobeam was broken. In addition, all $\mathrm{Ss}$ received intertrial goal placements following a further $30 \%$ of trials. A placement consisted of placing $S$ in the unbaited goal so that his body broke the photobeam. For control Ss the shock generator was then activated after a $.5-\mathrm{sec}$ delay and they received the same shock that IP Ss had received on punishment trials. Placements were administered $20 \mathrm{sec}$ af ter a running trial. The $S$ was then returned to the waiting stand for a further $20 \mathrm{sec}$ before being run on the next trial. Nonreward and nonreward-punishment trials were administered on a random schedule. The particular intertrial interval during which placement occurred was independently and randomly scheduled.

When treatment was complete, continuous punishment trials were carried out for 2 days. Each response was followed by a .25-mA shock and reward was withheld on each trial. If $S$ failed to respond within $180 \mathrm{sec}$ he was removed from the apparatus and assigned a response time of $180 \mathrm{sec}$.
RESULTS AND DISCUSSION

The response times of IP and control Ss were essentially similar during training. With the onset of treatment, IP Ss showed a marked increase in latency, reaching a mean of $40 \mathrm{sec}$ by Day 6 and showing a steady decline until the end of treatment. Throughout treatment, controls performed at their terminal training level and failed to show any effect of placement shock. At the end of treatment, IP Ss were running slower than controls $(6.4 \mathrm{sec}$ vs $1.7 \mathrm{sec})$ but with the onset of $\mathrm{CP}$ there was a crossover between the groups by Trial 4 . Throughout $\mathrm{CP}$ both groups showed a marked slowing in performance but controls responded more slowly than IP Ss $(99.2 \mathrm{sec}$ vs $46.6 \mathrm{sec}$, respectively). The difference between the groups was significant $[F(1,16)=17.12$, $p<.001]$. The main effect of trials was likewise significant $[F(19,323)=8.79$, $\mathrm{p}<.001]$, as was the Groups by Trials interaction effect $[\mathrm{F}(19,302)=4.97$, $\mathrm{p}<.001]$.

The results show that when frequency, intensity, duration, and locus of previous shock experience are held constant for IP and control Ss, the IPE continues to be observed. This result argues against a simple adaptation approach to the IPE and lends support to a learning-based interpretation of the effect. Since the only difference between the groups was the fact that IP Ss ran to shock while controls were placed for shock, a conflict-resolution model appears most appropriate. Thus an explanation of the IPE emphasizing the importance of responding in the presence of fear such as that offered by Miller (1960) or that derived from an extension of Amsel's (1962) frustration theory (Banks, 1966a) seems applicable.

\section{REFERENCES}

AMSEL, A. Frustrative nonreward in partial reinforcement and discrimination learning: Some recent history and a theoretical extension. Psychological Review, 1962,69, 306-328.

BANKS, R. K. Persistence to continuous punishment following intermittent punishment training. Journal of Experimental Psychology, 1966a, 71, 373-377.

BANKS, R. K. Persistence to continuous punishment and nonreward following training with intermittent punishment and nonreward. Psychonomic Science, 1966b, 5, 105-106.

BROWN, R. T., \& WAGNER, A. R. Resistance to punishment and extinction following training with shock or nonreinforcement. Journal of Experimental Psychology, 1964, 68, 503-507.

MILLER, N. E. Learning resistance to pain and fear: Effects of overlearning exposure and rewarded exposure in context. Journal of Experimental Psychology, 1960, 10, 137-145.

TERRIS, W., \& WECHKIN, S. Learning to resist the effects of punishment. Psychonomic Science, 1967,7, 169-170. NOTE

1. This research was supported in part by Grant No. APA 102 to the second author from the National Research Council of Canada. 